\title{
EDITORIAL
}

\section{Intensive Care Unit: Bangladesh Perspective}

Critical care or intensive care medicine is often thought to have begun in 1953 when Danish patients with poliomyelitis received invasive mechanical ventilation. ${ }^{1}$ although areas of some hospitals had been designated for patients recovering from anaesthesia or traumatic injuries a century earlier. ${ }^{2}$ These patients received care in a common location with intensified nursing support and manual ventilation provided by students and mortality declined. Intensive care units (ICUs) subsequently became a crucial component of hospital care. ${ }^{1}$

In the subject of Anesthesia, we have the following specialities like Anaesthesia, Analgesia or painmanagement, CPR, ICU (Intensive Care Unit) and Palliative care Medicine, among which Palliative care Intensive Care medicine are very much up growing. After the first establishment of ICU in 1953 for treating patient of poliomyelitis, the modern concept of ICU is developed in different subspecialty like CCU,Neuro ICU, Obstetric ICU, Neonatal ICU, Trauma Burn \& Poisoning ICU. Overtime the increasing interest in the ICU led to demands in highly academic activities specially for a post graduate course curriculum, research into pathophysiological processes not only with patients, but also with highly equipped ventilators, many invasive procedures, crossinfection, VAP (Ventilatory Assisted Pneumonia), treatment regimens, outcome of patient, Journal, training, short and long term course curriculum, etc.

An intensive care unit (ICU) has been defined as a specially staffed, specially equipped, separate section of a hospital dedicated to the observation, care, and treatment of patients with life-threatening or potentially life-threatening illnesses, injuries, or complications, from which recovery is possible ${ }^{3}$. From the beginning till the present day, ICU is one of the main subspeciality in the subject of Anaesthesiology but involves all specialities of medical science like surgical/medical internist, all subspecialty specially cardiologist, pulmonologist, physicist, psychiatrist, nursing staff, or any acute, casualty or emergency discipline.
Patients in an ICU generally fall into three main categories: those with acute organ dysfunction (including those whose ultimate outcome is unclear and thus receive long-term intensive organ support), those who have undergone a major procedure and are monitored in the peri-intervention period to prevent and detect acute organ dysfunction, and those whose trial of intensive care has failed and are receiving end-oflife care. Intensive care is delivered by an interdisciplinary team that includes not only nurses and physicians, but also respiratory therapists, physical and occupational therapists, biotechnicians, pharmacists, nutritionists, social workers, and spiritual care providers.

The first ICU in Bangladesh was established in the year of 1980 at National Institute of Cardiovascular disease (NICVD). Only two Govt. Medical college Hospital at Dhaka and Chittagong have ICU. Number of ICU is increasing steadily in recent years. Unfortunately most of them are concentrated in Dhaka.Currently there are about fifty ICUs with five hundred \& fifty beds.Among them, most of the ICU is located within the private sector. $^{4}$

The number of beds of ICU usually range from 1-4 per 100 beds, but less than 6 beds and more than 24 beds is not ideal because lower number of beds will not be cost effective \& more beds may be difficult to manage. If at least $5 \%$ of all beds were dedicated for ICU care, then in Bangladesh we need at least 2500 ICU beds assuming that currently we have approx. 50 thousand hospital beds in the whole country.

In our private sector, almost all ICU function as a mean of generating more money. So before setting up an ICU, the type, size, site, and designing of the ICU is very important. In perspect ${ }^{2}$ of Bangladesh, development of multidisciplinary ICU which supports all type of critically ill patients is managed by the Anaesthesiology department with the aid of specialist intensivists. But paediatric, neonatal, infectious disease patients \& burn patients should have an individual ICU. 
ICU consultant in charge will supervise the patients every moment and will contact other specialists according to the need of the patient. ICU should be designed by the health planning policy maker, hospital planning experts, and all types of instrument specialists or their management department.

Ideally, the ICUs have to be on the ground floor and not far from the casualty department and a generalward ${ }^{5}$. As recommended by Miranda et al the supporting services such as an operating theatre, the department of radiology, the laboratories, and the blood bank should be nearby. ${ }^{6}$ On the contrary, in Bangladesh it has been found that the ICUs are surrounded by random facilities and services. There does not seem to be any standard practice or design. Because of the unavailabilityof a standard location and easy pathway to reach the ICU, the patients and their relatives have to search for it, which results in delays and disturbance to other patients.

ICU is an extremely costly unit with about $50 \%$ fruitful outcome. The average cost of ICU in Bangladesh can range from Taka 15000 per day to as high as taka 60,000 per day in the private sectors hospital. Institute like BSMMU treat patients on subsidy but not on free basis.It has been observed that increased ICU beds in private sectors does not ensure increased health care delivery for the general population where average daily per capita income is taka 100 per day. Existing ICU in our country with the of exception of few are neither adequately staffed in terms of trained doctors and critical care nurse nor adequately equipped.

In 2007, one study conducted by Critical Care Medicine,BIRDEM hospital showed that there were one ICU bed for every 100 general beds in Bangladesh. It also showed that $95 \%$ ICUs were located in the city of Dhaka and $60 \%$ of ICU beds had accompanying mechanical ventilators. Arterial blood gas analyzer, bedside echocardiography and ultrasonography facilities were available in $70 \%, 55 \%$ and $65 \%$ unit respectively. This study also showed that $51 \%$ of doctors and $36 \%$ of nurses were cardiopulmonary resuscitation trained.

Human resource development is very important part of ICU.Several retrospective studies have shown better patient outcome and cost benefits when ICU patient are managed in closed units where all patients are cared for one team of intensivists in collaboration with other primary care services. ${ }^{7,8}$
Currently MD course on Critical Care Medicine(CCM) is running at Dhaka Medical College and BIRDEM Hospital. It is imperative that postgraduate medical student of other discipline like Internal Medicine, Cardiology, Pulmonology, Surgery and Anaesthesiology need to have mandatory exposures in CCM. Whereas Bangladesh need at least five hundred qualified postgraduate Intensivists at the moment, Our first postgraduate critical care medicine specialists is expected to roll down our MD courses not earlier than 2012.

So advances in organizationof ICU have led to reduction in the mortality rate and morbidity suffered by the severely critically ill patient. It is high time for this development of clinical protocols and course curriculum to be done by the Anaesthesiology department (like in the UK). Many of our govt. medical colleges has an ICU department which lack of so many things which we are all aware of increasing demand of ICU, nurse, and other human staff with good equipment can improve the quality of care of the patient.

The young specialty of intensive care has evolved from its origins in treatment of poliomyelitis victims with respiratory failure, to an interdisciplinary team that caresfor patients in an ICU, and to a broader mandate to treat critically ill patients irrespective of geographical location or use of specific technology. Intensive care capacity is scarce in the developing world; efforts to improve the care of the critically ill in these settings are emerging.

ICU is highly expensive, has a rapid turnover, and needs highly skilled personnel with standard equipment's. Budget education, training of all personnel working in the ICU, postgraduate course and research is important. In digital Bangladesh successful spread of ICU information in medical colleges and hospitals will improve the clinical environment. But ICU personnel related factors, disturbed electronic database may interfere with the wide spread distribution, though we will proceed for the development of rational use of computerized protocol in the multidisciplinary intensive care unit.

(J Bangladesh Coll Phys Surg 2012; 30: 187-189)

\footnotetext{
Professor Dr. U.H. Shahera Khatuna

Dr. Md. Wakely Mandal ${ }^{b}$

a. Professor of Anaesthesiology \& ICU, Dhaka Community Medical College, Dhaka.

b. Assistant Professor of Anaesthesiology, Shaheed Monsur Ali Medical College, Uttara, Dhaka.
} 


\section{Reference}

1. Adhikari NKJ, Fowler RA, Bhagwanjee S, Rubenfeld GD. Critical care and the global burden of critical illness in adults. Lancet 2010; 375: 1339-46.

2. Grenvik A, Pinsky MR. Evolution of the intensive care unit as aclinical center and critical care medicine as a discipline.Crit Care Clin2009; 25: 239-50.

3. George A Skowronski, Bed rationing and allocation in the intensive care unit, Current Opinion in Critical Care 2001, 7:480-484

4. Faruq MO, Ahsan ASMA, Fatema K, Ahmed F, Sultana A, ChowdhuryRH.An audit of Intensive care services in Bangladesh. Ibrahim Medical college journal 2010;4:13-15
5. ICU in changeover mode, Healthcare ManagementExpress; Issue - 15-31; August 2003.

6. Bhutkar G, Katre D, Rajhans N, Deshmukh S. Scope of Ergonomics Design and Usability for anIntensive Care Unit (ICU): An Indian Perspective HFESA Journal, Ergonomics Australia 2008; 22:26-32

7. Manhous CA, Amoateng-Adjepong Y, Al-Kharfat T, Jacob B,Alnuaimat HM, Chatila N, et al. Effect of medical intensivist on patient care in a community teaching hospital. Mayo clinic Proceedings 1997;72:391-399

8. William III C, Dentschman CS, Anderson III HL, Relly PM, Behringer EC, Schnab CW et al. Effect of an organized critical care service on outcome and resource utilization: a cohort study. Critical care Medicine 1999;27: 270-274. 